\section{Arco de intrusión de Burstone para la corrección de la mordida profunda. Reporte de caso}

\section{Burstone's intrusion arch for deep overbite correction. Case report}

\begin{abstract}
Resumen
La mordida profunda se define como una maloclusión vertical, donde existe un incremento del sobrepase vertical de los incisivos superiores sobre los incisivos inferiores, puede deberse a una supraerupción de los dientes anterosuperiores, incremento de la curva de Spee o una combinación de ambos. Se presenta un caso de una paciente de 23 años de edad, con perfil convexo, sobremordida aumentada y retroinclinación de los incisivos centrales superiores. Se empleó el arco de intrusión propuesto por Burstone para la corrección simultanea de la supraerupción y retroinclinación de los incisivos superiores, logrando una oclusión estable tras un seguimiento de dos años. Este caso permitió demostrar la aplicación de los conceptos biomecánicos propuestos por Burstone para el tratamiento de la mordida profunda.
\end{abstract}

Palabras clave: Maloclusión; Ortodoncia correctiva; Sobremordida (fuente: DeCS Bireme).

\section{Caso Clínico}

Marco Antonio Sánchez-Tito 1,a

${ }^{1}$ Universidad Privada de Tacna, Facultad de Ciencias de la Salud. Tacna, Perú.

${ }^{a}$ Especialista en Ortodoncia y Ortopedia Maxilar.

\section{Correspondencia:}

Marco A. Sánchez-Tito

Correo electrónico: marcosanchez2183@gmail.com Capanique s/n, Pocollay, Facultad de Ciencias de la Salud, Universidad Privada de Tacna, Tacna - Perú.

\section{Editora:}

Sandra Patricia Palomino-Gómez

Universidad Nacional Mayor de San Marcos, Perú.

Conflicto de intereses: $\sin$ conflicto de intereses.

Fuente de financiamiento: autofinanciado.

Recibido: $18 / 04 / 19$

Aceptado: 07/07/19

Publicado: 12/09/19

\begin{abstract}
Deep overbite is defined as a vertical malocclusion, where there is an increase of the overlap of the upper incisors over the lower incisors, it may be due to supraeruption of the anterior upper teeth, increase of the curve of Spee or a combination of both. We present a case of a 23 years old patient, with a convex profile, increased overbite and retroinclination of the central upper incisors. A Burstone's intrusion arch was used for simultaneous correction of the supraeruption and retroinclination of the upper incisors, achiving a stable occlusion after two years of follow-up. This case allows to demonstrate the application of biomechanical concepts proposed by Burstone for the treatment of deep bite malocclusion.
\end{abstract}

Keywords: Malocclusion; Orthodontics, corrective; Overbite (source: MeSH NLM). 


\section{Introducción}

La mordida profunda es una maloclusión vertical que presenta una etiología multifactorial, y se define como una condición clínica donde los incisivos superiores recubren de manera exagerada a los incisivos inferiores ${ }^{1,2}$, considerando que una sobremordida dentro de los patrones normales se aproxima a $3 \mathrm{~mm}$ o $30 \%$, valores mayores son considerados como mordida profunda ${ }^{2}$. En términos generales la mordida profunda es un conjunto de características que involucran una alteración esquelética, dentaria y muscular ${ }^{3,4}$. La etiología de la mordida profunda puede estar relacionada a alteraciones en el crecimiento de la maxila y/o la mandíbula, alternaciones en la función de los labios y lengua, pero principalmente a alternaciones a nivel dentoalveolar; así en los casos de mordida profunda sin compromiso esquelético se puede observar la supraerupción de los incisivos superiores y/o inferiores 5 . En estos casos la mordida profunda es un síntoma y por lo tanto pueden existir muchas formas para corregirlas, así en los casos con supraerupción de los incisivos estará indicada la intrusión de los mismos mientras que en aquellos pacientes que presenten infraerupción del segmento posterior estará indicada la extrusión ${ }^{6}$.

Burstone ${ }^{7}$ introdujo en 1977 una técnica de intrusión de los dientes anteriores como una opción para la corrección de la mordida profunda. Este arco de intrusión está constituido por tres partes: a) una unidad de anclaje posterior, que son segmentos de alambres rígidos que unen los molares con los premolares y/o caninos y el empleo de un arco transpalatino, esto con la intención de actuar como unidad de anclaje; b) un segmento an- terior, se trata de un segmento de alambre rígido que se inserta en el slot de los brackets sobre los dientes que serán intruidos, y c) el arco de intrusión.

El arco de intrusión se basa en el principio de un cantiléver, donde se aplican fuerzas únicas sin la presencia de un momento resultante en los incisivos. Este arco de intrusión es construido con alambre rectangular de aleación de titanio molibdeno (TMA) de 0,017 " x 0,025". El arco se activa de tal forma que la porción incisal quede alejada de la región gingival de los incisivos centrales, cuando es amarrado al segmento anterior de alambre se genera una fuerza intrusiva. En cuanto a la magnitud de fuerza aplicada por el arco de intrusión, Burstone recomienda que en los casos donde se requiera la intrusión de los cuatro incisivos la magnitud de fuerza no debe exceder de $80 \mathrm{~g}$, variaciones en la magnitud de la fuerza aplicada están relacionadas con el tamaño de las raíces y otras consideraciones biológicas, así como de consideraciones mecánicas como la forma, sección y el material con el que es construido el arco de intrusión. Por otro lado, se debe considerar la consistencia de la fuerza aplicada, esto se traduce en la razón fuerza/deflexión $(F / \Delta)$; una razón baja de $F / \Delta$ tiene muchas ventajas, como generar fuerzas relativamente constantes, requerir pequeñas activaciones y mayor tiempo entre ellas ${ }^{6,8}$ (Figura 1).

Se deben tener consideraciones respecto al punto de aplicación de la fuerza, el arco de intrusión no se debe colocar en el slot de los brackets si lo que se quiere es evitar los efectos adversos de segundo y tercer orden, como las inclinaciones laterales de las raíces, generando pérdida del paralelismo radicular y la inclusión de torques desfavorables a los dientes. Para evitar estos efectos

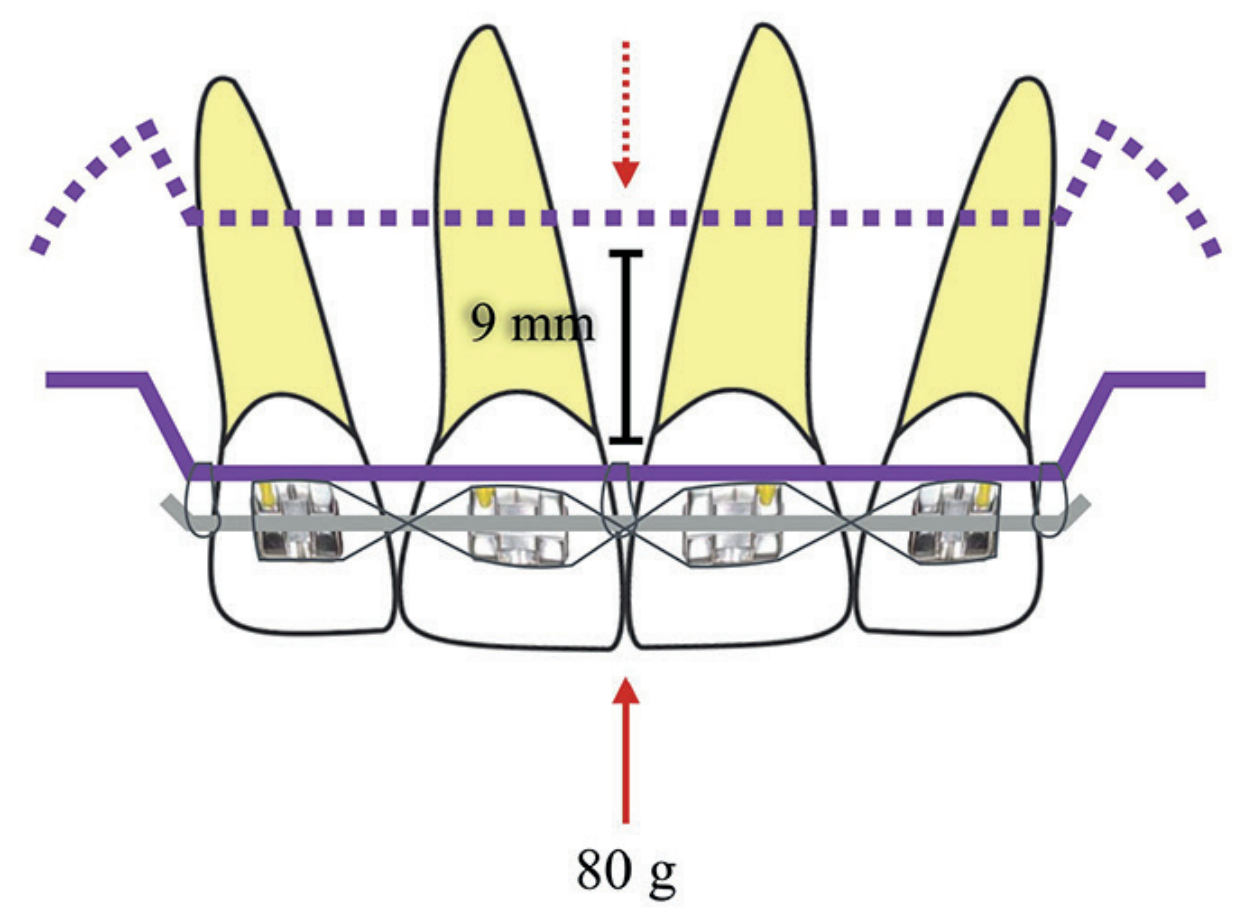

Figura 1. Esquema ilustrando parte del arco de Intrusión. El promedio de activación del arco de intrusión es determinado por el promedio de fuerza requerido. Se debe considerar además la razón $F / \Delta$. Una activación del arco de intrusión de 9 $\mathrm{mm}$ generará una fuerza de $80 \mathrm{~g}$ 
adversos se emplea el segmento de alambre rígido insertado en el slot de los brackets de los dientes anteriores, y el arco de intrusión es atado en un solo punto a este segmento, de esta manera el sistema de fuerzas aplicado es predecible, lo que se conoce como un sistema de fuerzas estáticamente determinado ${ }^{9,10}$. Identificar el centro de resistencia (CR) de los incisivos es importante para decidir donde realizar la aplicación de la fuerza. Si el arco de intrusión se ata en mesial de los incisivos centrales, el sistema de fuerzas generará una intrusión mientras el momento causará una inclinación anterior de la corona y un movimiento lingual de las raíces (Figura 2). Este sistema de fuerzas no es completamente efectivo, sin embargos en algunos casos como en las maloclusiones Clase II división 2, éste será un movimiento completamente favorable para los incisivos centrales, ya que ayudará a corregir las inclinaciones de estos dientes. Si el requerimiento es de una intrusión pura, el arco deberá ser atado por distal a los incisivos, así la línea de acción de la fuerza pasa a través del CR de los incisivos, la intrusión se hace más efectiva debido a que no se producen efectos adversos por el momento generado ${ }^{6,9,11}$.

El presente reporte de caso tiene por objetivo demostrar el uso del arco de intrusión de Burstone para la corrección de una mordida profunda con retroinclinación de los incisivos centrales superiores.

\section{Reporte del caso}

Diagnóstico. Paciente femenina de 21 años de edad, acude a la consulta solicitando alternativa de tratamiento ortodóntico para mejorar la exposición de su sonrisa y resolver el apiñamiento dentario inferior.

En el examen clínico extraoral, presentó patrón facial simétrico, sonrisa gingival con desviación de la línea media dentaria hacia el lado derecho, perfil convexo moderado, se observa buena exposición del tercio me- dio y buen ángulo labial. Al examen intraoral se observa presencia de defecto de la superficie de esmalte en las piezas 2.1 y 3.2, relaciones molares de Clase I, relación canina derecha de Clase I y relación canina izquierda con tendencia a Clase II. Los incisivos superiores se encontraban retroinclinados (Figura 3).

En el análisis de modelos se observó una mordida profunda y una curva de Spee pronunciada, un overjet de 1 $\mathrm{mm}$ y una discrepancia dentoalveolar inferior de $-4 \mathrm{~mm}$ (Figura 4). En el análisis cefalométrico se observa un patrón esquelético de Clase II $\left(\mathrm{ANB}=6^{\circ}\right)$ con retroinclinación de los incisivos superiores (I.NA $=3^{\circ}$, I. $I=153^{\circ}$ ), como puede ser observado en la Figura 5 y la Tabla. La radiografía panorámica muestra la presencia de todos los dientes, incluido los terceros molares (Figura 6).

Objetivos de tratamiento. Los objetivos de tratamiento fueron la corrección de la mordida profunda, la nivelación de la curva de Spee, corrección del apiñamiento anteroinferior, y corrección de las inclinaciones de los incisivos superiores; mejorar la exposición de los incisivos por medio de la corrección de la línea media superior dentaria respecto de la línea media facial.

Evolución del tratamiento. Se instalaron brackets metálicos convencionales prescripción Roth slot 0,022 " x 0,0028 " y se colocaron bandas en los primeros molares superiores e inferiores, se soldaron tubos simples y tubos linguales para la instalación de un arco transpalatino. Para la corrección de la mordida profunda, en la arcada superior se confeccionó un arco de intrusión de Burstone con alambre TMA de 0,017" x 0,025" que alcance el slot de los brackets de los incisivos centrales, se eligió el arco de intrusión de Burstone debido al potencial de corrección simultaneo de la sobremordida y de la retroinclinación de los incisivos centrales, la fuerza generada por la activación del arco de intrusión fue medida con un dinamómetro de tal forma que fue-
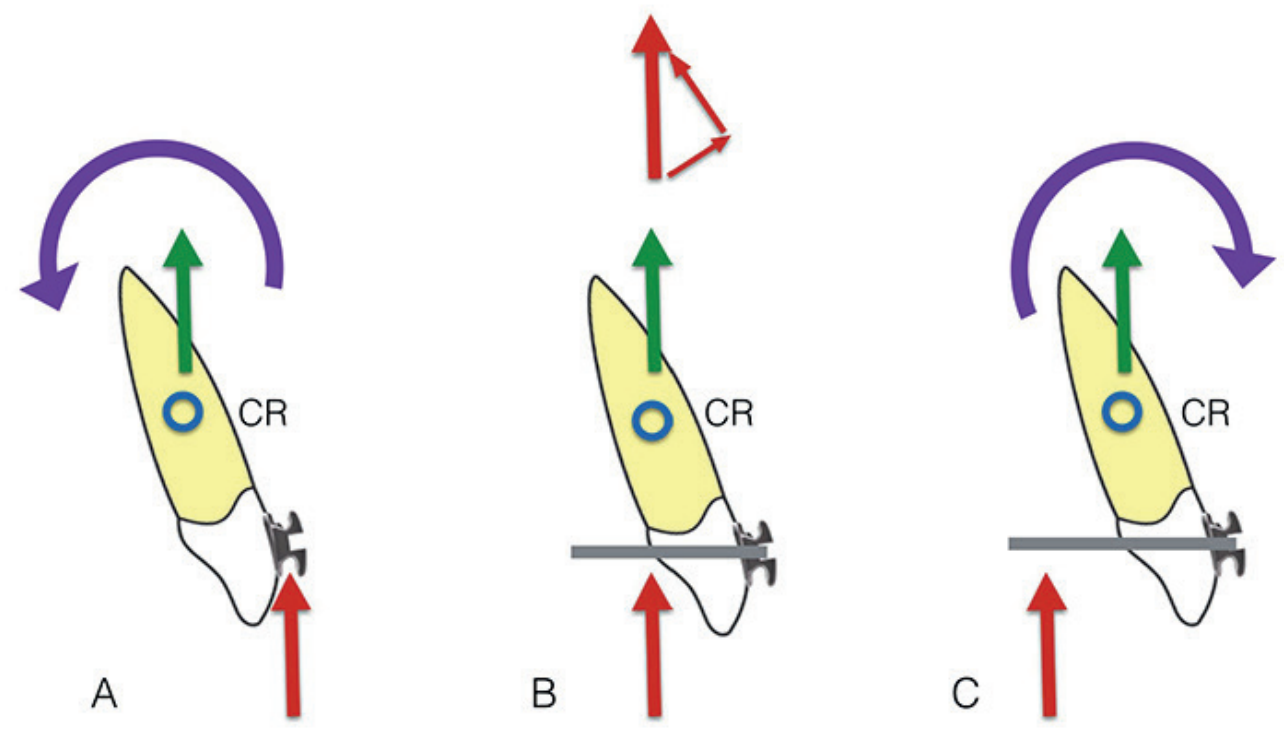

Figura 2. Cambio del punto de aplicación de la fuerza respecto al centro de resistencia (CR). A. Aplicación por delante del CR generará inclinación vestibular de la corona. B. Aplicación a nivel del CR generará intrusión verdadera. C. Aplicación distal al CR producirá una inclinación hacia palatino de la corona 

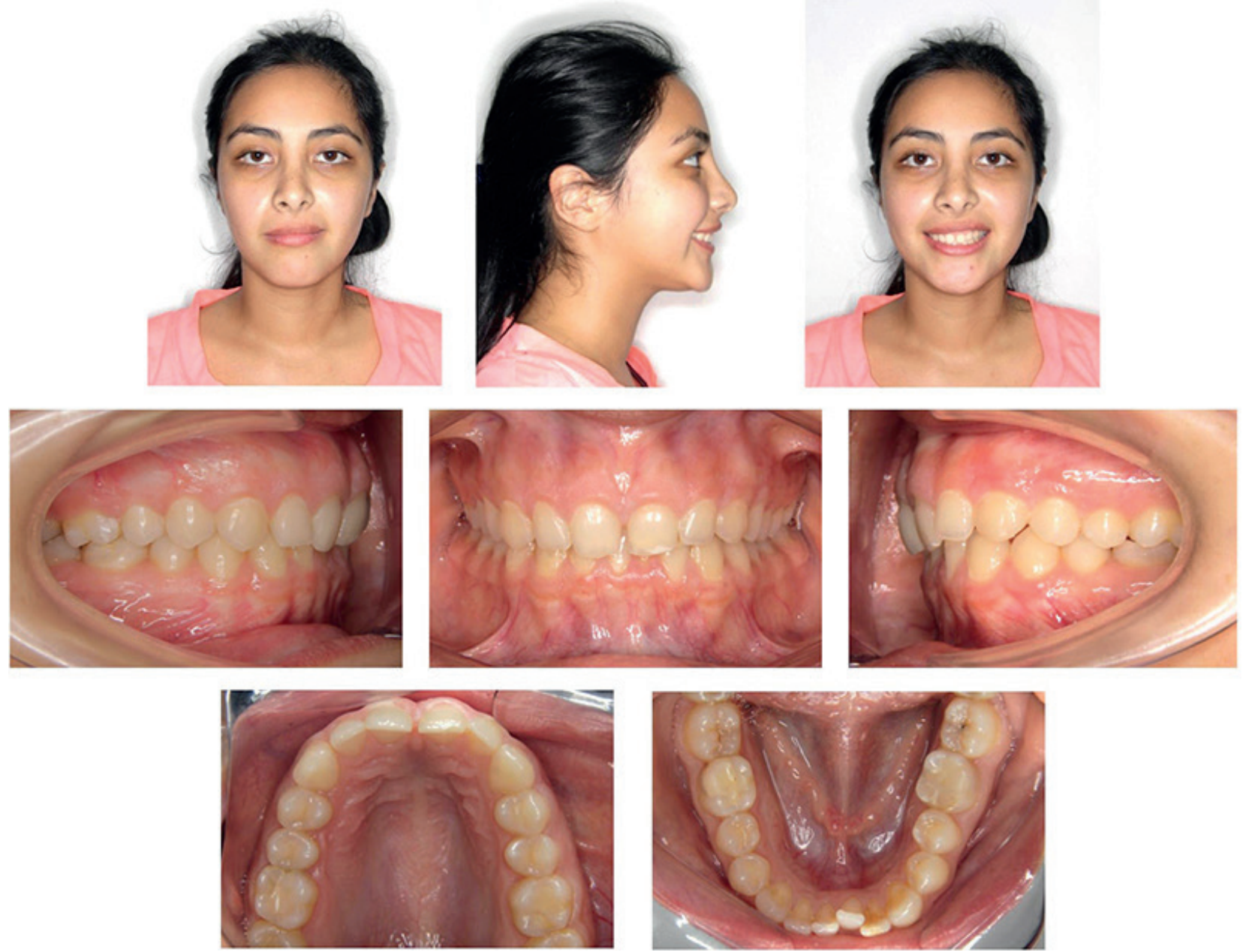

Figura 3. Fotografías extraorales e intraorales de inicio
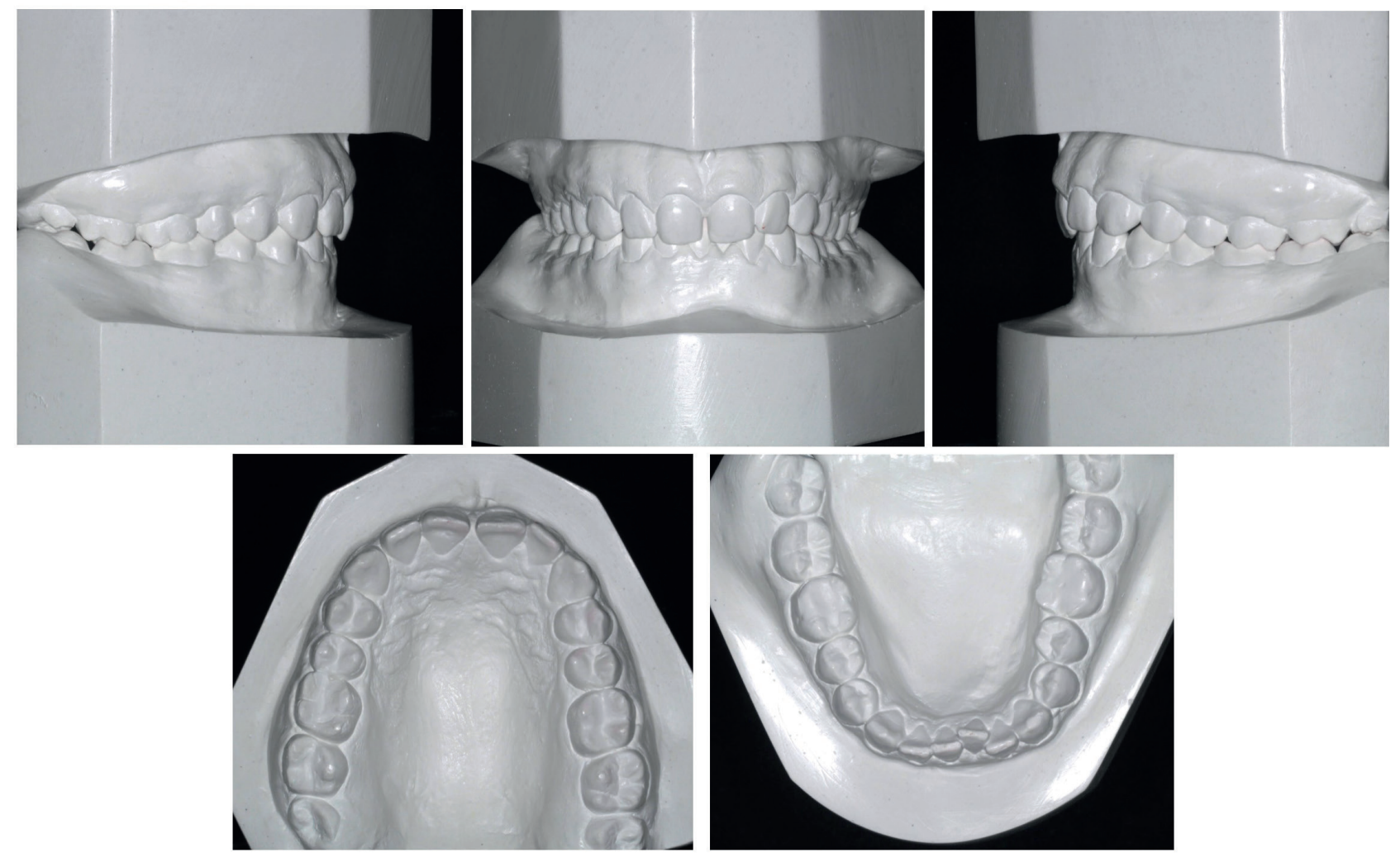

Figura 4. Modelos de estudio de inicio 

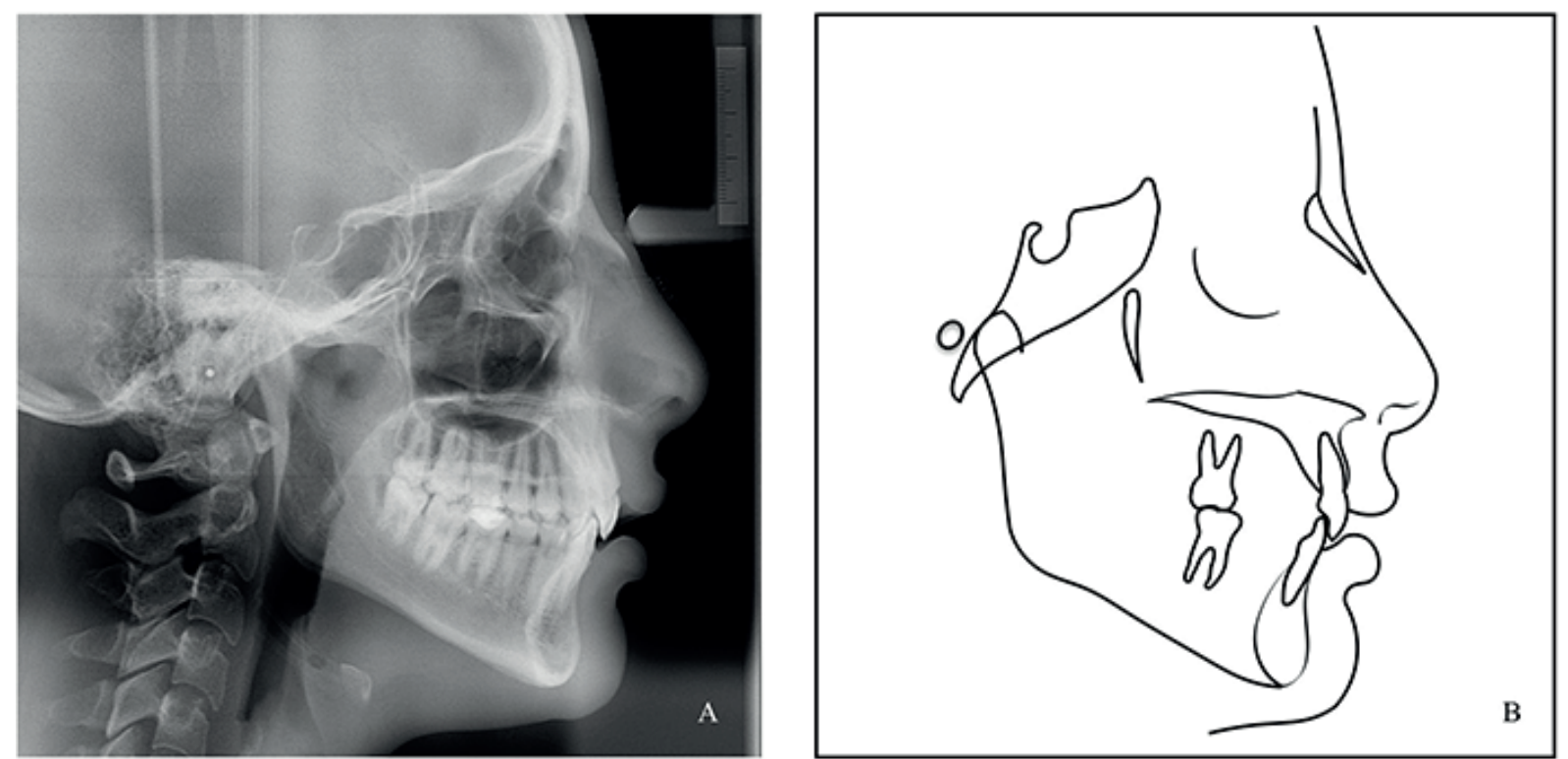

Figura 5. A. Radiografía cefalométrica de inicio. B. Trazado cefalométrico

Tabla. Medidas cefalométricas

\begin{tabular}{|c|c|c|c|c|c|}
\hline \multirow{8}{*}{ Patrón esquelético } & SNA & (Steiner) & $\begin{array}{c}82^{\circ} \\
\text { (Norma) } \\
\end{array}$ & $\begin{array}{c}84^{\circ} \\
\text { Inicio }\end{array}$ & $\begin{array}{c}86^{\circ} \\
\text { Final } \\
\end{array}$ \\
\hline & SNB & (Steiner) & $80^{\circ}$ & $78^{\circ}$ & $80^{\circ}$ \\
\hline & ANB & (Steiner) & $2^{\circ}$ & $6^{\circ}$ & $6^{\circ}$ \\
\hline & Ángulo de la convexidad & (Downs) & $0^{\circ}$ & $15^{\circ}$ & $14^{\circ}$ \\
\hline & Eje $Y$ & (Downs) & $59^{\circ}$ & $61^{\circ}$ & $60^{\circ}$ \\
\hline & Angulo Facial & (Downs) & $87^{\circ}$ & $88^{\circ}$ & $88^{\circ}$ \\
\hline & Sn-GOGn & (Steiner) & $32^{\circ}$ & $37^{\circ}$ & $38^{\circ}$ \\
\hline & FMA & (Tweed) & $25^{\circ}$ & $27^{\circ}$ & $25^{\circ}$ \\
\hline \multirow{7}{*}{ Patrón dental } & IMPA & (Tweed) & $90^{\circ}$ & $85^{\circ}$ & $96^{\circ}$ \\
\hline & 1.NA & (Steiner) & $22^{\circ}$ & $3^{\circ}$ & $14^{\circ}$ \\
\hline & $1-\mathrm{NA}$ & (Steiner) & $3 \mathrm{~mm}$ & $-3 \mathrm{~mm}$ & $0 \mathrm{~mm}$ \\
\hline & 1.NB & (Steiner) & $25^{\circ}$ & $21^{\circ}$ & $32^{\circ}$ \\
\hline & $1-\mathrm{NB}$ & (Steiner) & $4 \mathrm{~mm}$ & $3 \mathrm{~mm}$ & $4^{\circ}$ \\
\hline & 1.1 & (Downs) & $130^{\circ}$ & $153^{\circ}$ & $128^{\circ}$ \\
\hline & 1-APo & (Ricketts) & $1 \mathrm{~mm}$ & $-1 \mathrm{~mm}$ & $1 \mathrm{~mm}$ \\
\hline
\end{tabular}

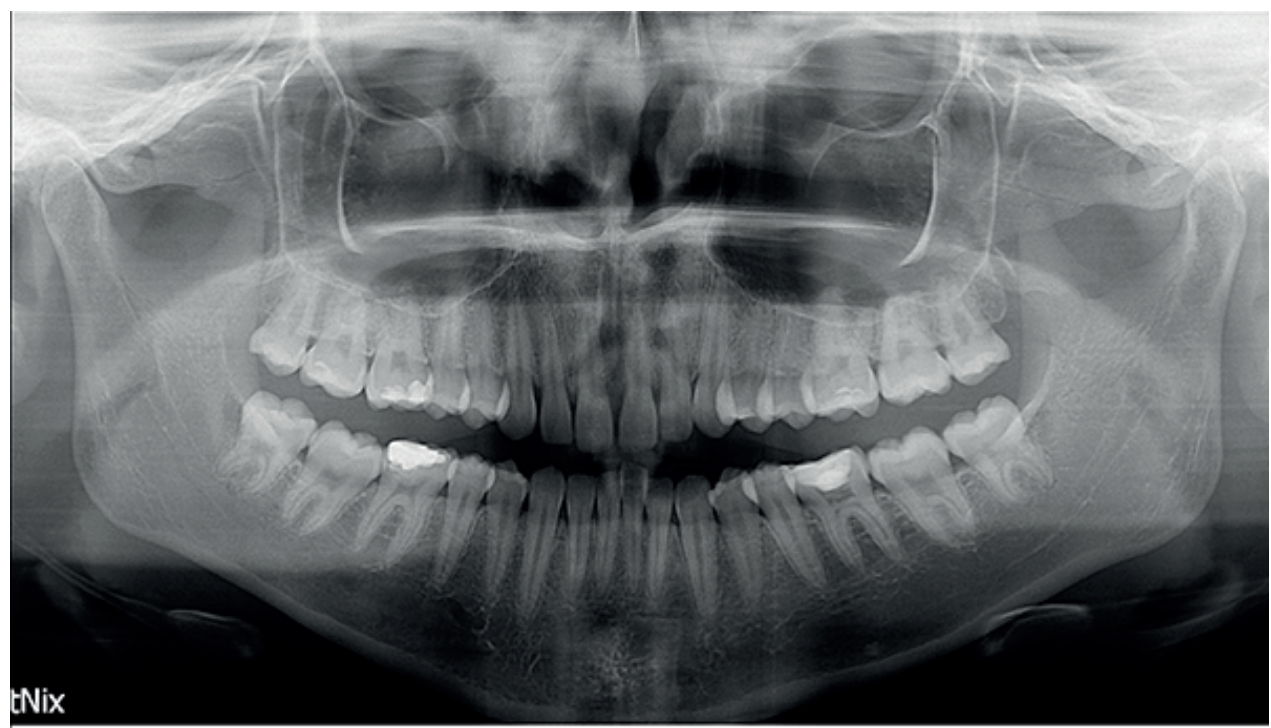

Figura 6. Radiografía panorámica de inicio 
se de 40 g. Se colocó un arco acero de 0,016” para el alineamiento del resto de los dientes superiores con un puente a nivel de los incisivos centrales para permitir la intrusión de los mismos al activar el arco de intrusión. $\mathrm{El}$ arco de intrusión se ató con alambre de ligadura por distal de los incisivos con la intención de generar intrusión y una ligera inclinación de los incisivos centrales superiores (Figura 7). En el arco inferior se colocó un arco Niti de 0,014" con un resorte abierto a nivel de la pieza 3.2, luego de abrir el espacio se incluyó a la pieza 3.2 en el tratamiento. Conseguida la intrusión de los incisivos centrales superiores, se colocó un arco Niti 0,016 " x 0,022 " para continuar con el alineamiento de los dientes. En este momento se evidenció una relación de Clase II canina del lado izquierdo. Se colocaron arcos de acero de 0,017 " x 0,025 " superior e inferior y se empezó a usar un elástico de 3/16" medio en disposición de Clase II para el lado izquierdo. Para el acabado se seccionó el arco superior y se confeccionó un segmento de alambre con activación para mejorar la relación canina del lado izquierdo asociado al uso de elástico de 3/16". Corregida la relación canina Clase II del lado izquierdo se colocó un arco de acero superior de 0,020 " con un doblez en "Z" para preservar la posición de la pieza 2.3 $y$ un arco de acero inferior de 0,017 x 0,025" (Figura 8).

Luego de obtener los resultados esperados, se colocó un aparato removible superior que fue usado como retenedor durante 1 año, por 24 horas al día; los siguientes 6 meses el retenedor fue usado solo por la noche, luego de este periodo de tiempo el uso del retenedor se indicó de manera alternada por las noches por 6 meses más. En el arco inferior se empleó un retenedor fijo confeccionado con alambre de acero de 0,012 " entorchado de canino a canino adherido con resina a la superficie lingual de los dientes.
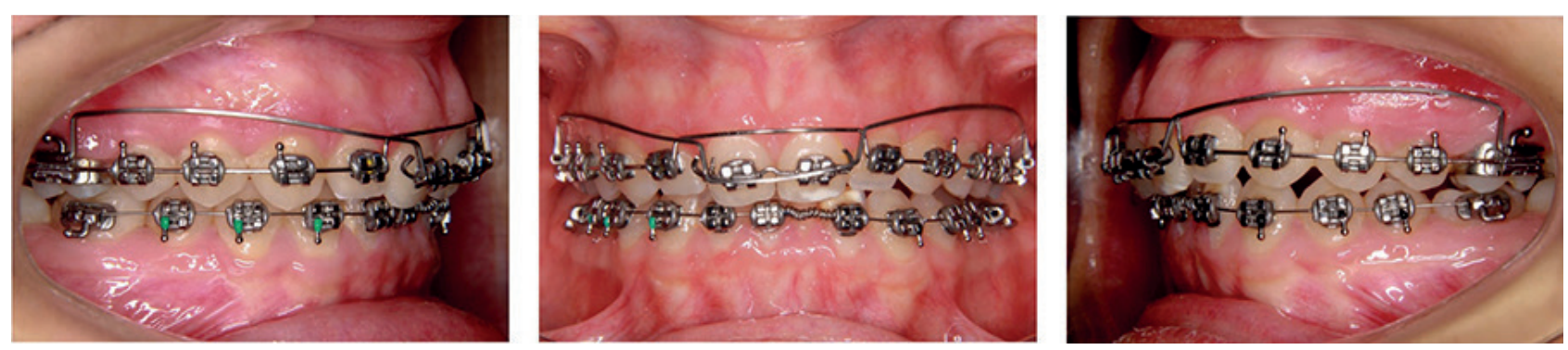

Figura 7. Arco de Intrusión fabricado en alambre TMA 0,017" x 0,025" y amarrado con alambre de ligadura por distal de los incisivos para generar intrusión asociada a inclinación de los incisivos
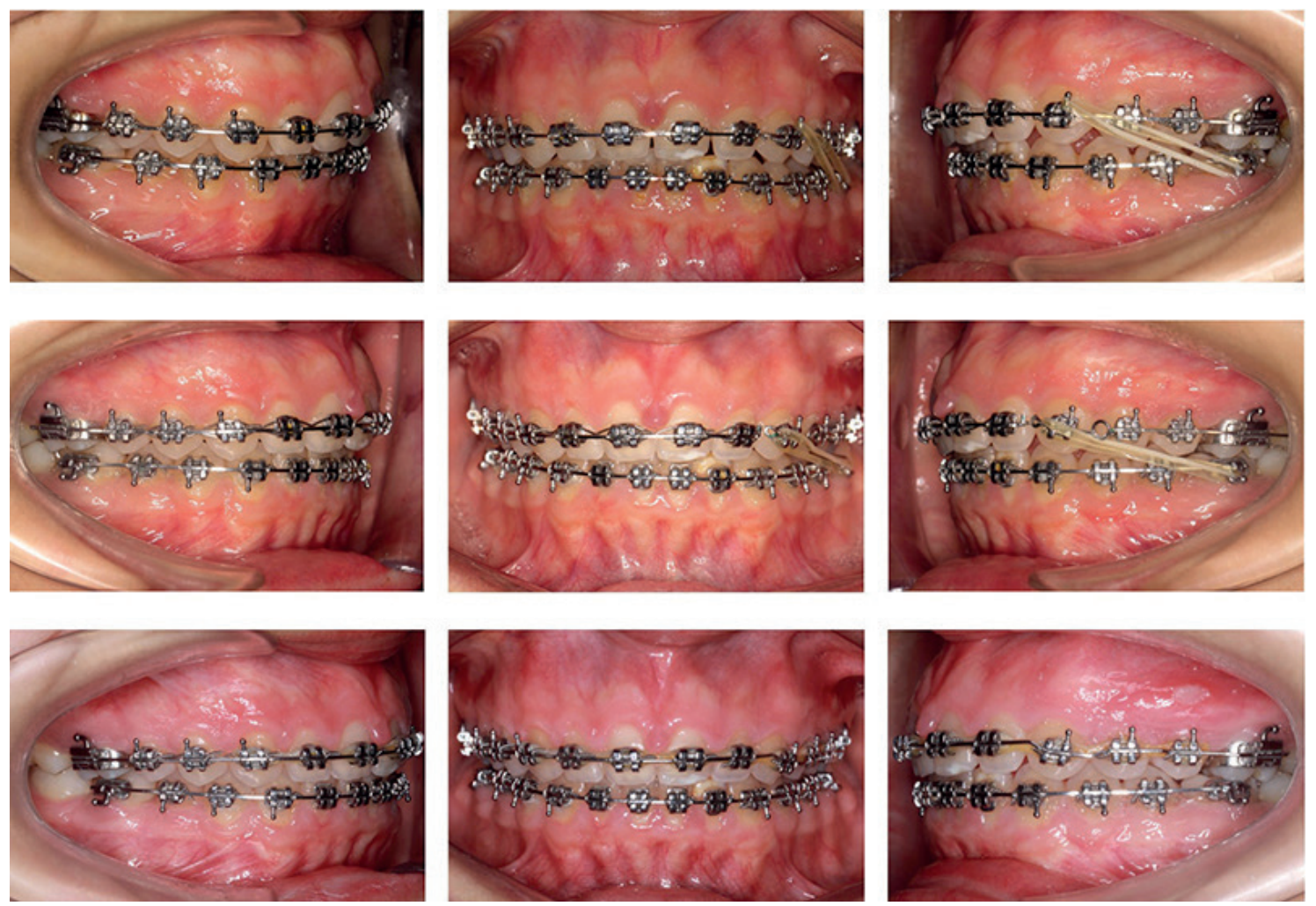

Figura 8. Uso de elásticos clase II para la corrección de la relación canina izquierda. Las fotografías de la primera fila muestran el inicio término de la fase de alineamiento con arcos de acero 0,017x0,025". La segunda fila muestra el uso de arco segmentado en el lado izquierdo activado para el control vertical durante el uso del elástico 3/16" de Clase II. La tercera fila muestra el uso de arco de acero 0,020 " con doblez en " $z$ " a nivel del canino izquierdo superior para mejorar el acoplamiento de la oclusión 
Resultados del tratamiento. Luego de 24 meses del tratamiento, los objetivos fueron alcanzados, obteniendo relaciones oclusales estables, mejorando la expresión de la sonrisa de la paciente. Se logró una posición correcta de los incisivos centrales superiores. Se consiguió correcciones adecuadas sobre la mordida profunda y la curva de Spee (Figuras 9, 10 y 11). Los valores finales de los factores cefalométricos se muestran en la Tabla; se mantuvo el ángulo ANB en $6^{\circ}$, una corrección del ángulo interincisivo de $153^{\circ}$ a $128^{\circ}$, con un incremento del IMPA de $85^{\circ}$ a $96^{\circ}$. En la Figura 12 se puede observar el trazado cefalométrico de superposición. La radiografía
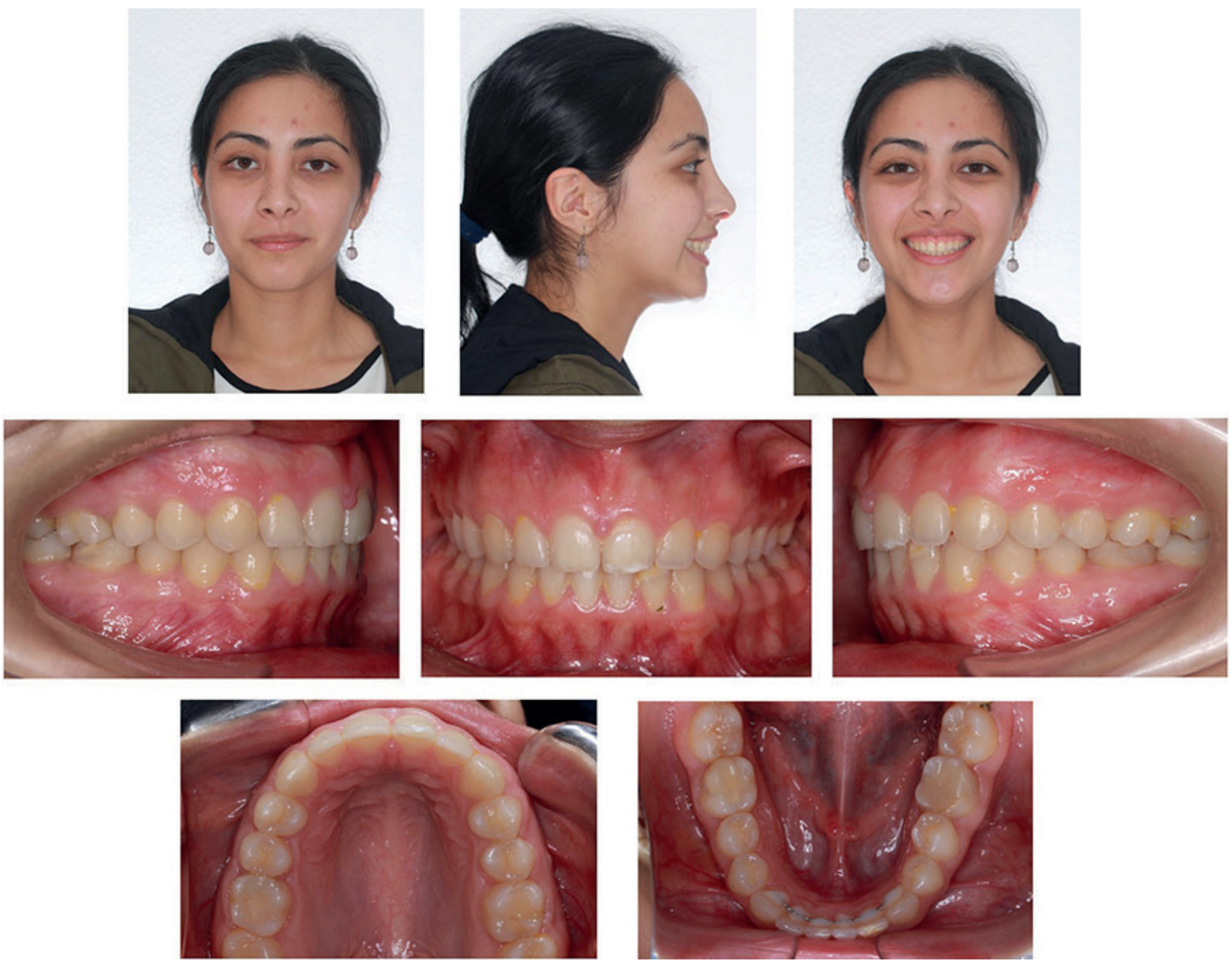

Figura 9. Fotografías extraorales e intraorales finales
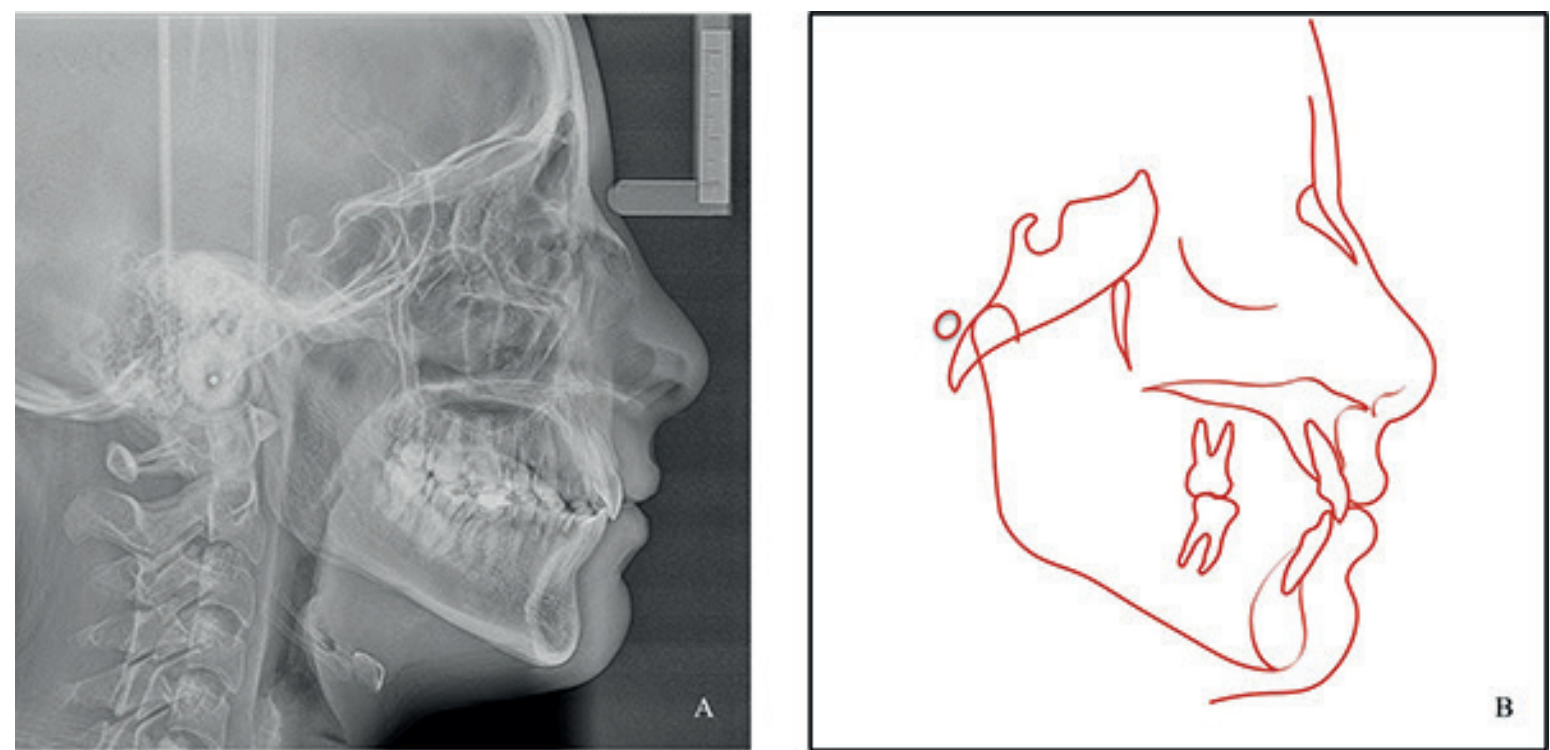

Figura 10. A. Radiografía cefalométrica final. B. Trazado cefalométrico 

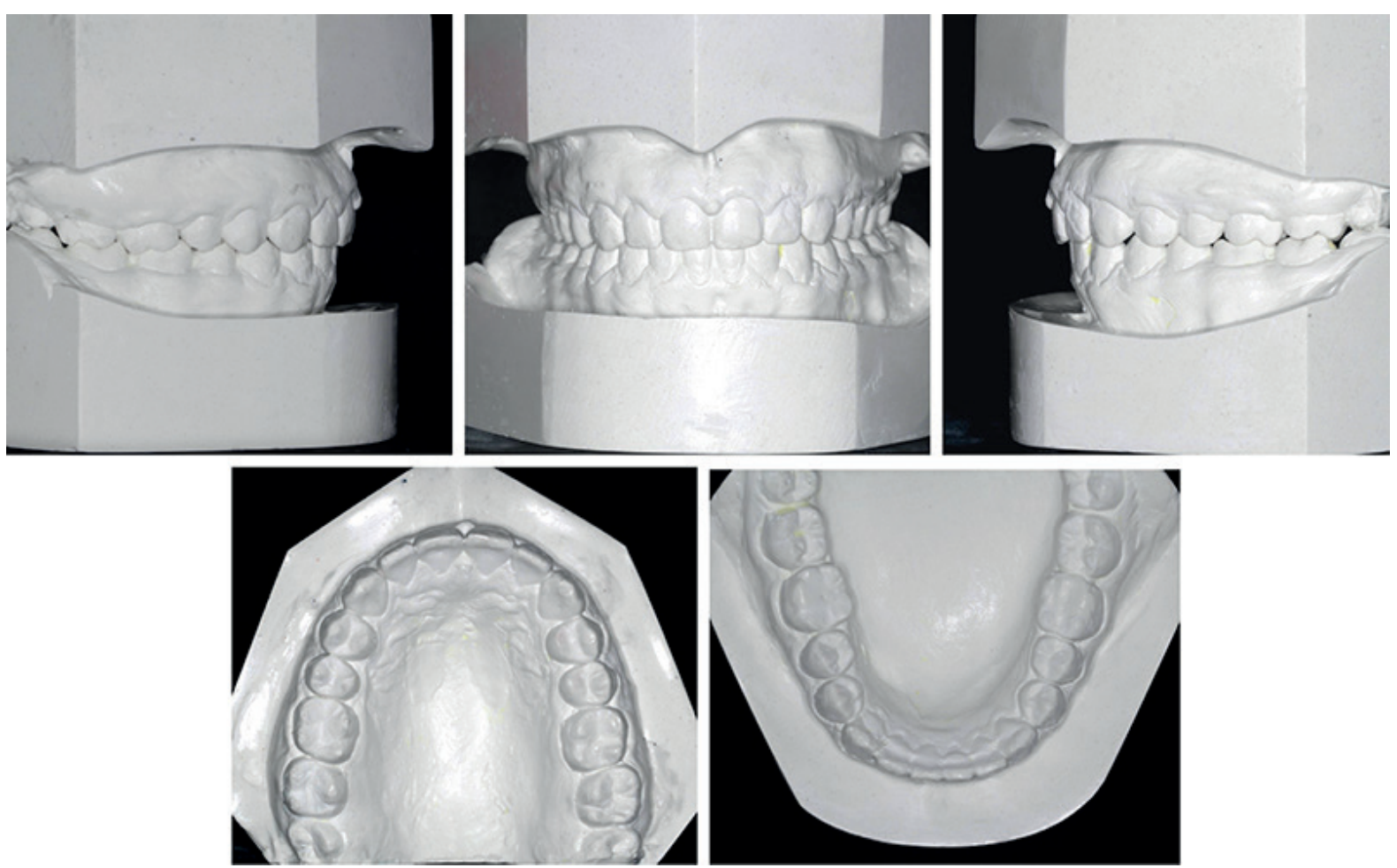

Figura 11. Modelos finales
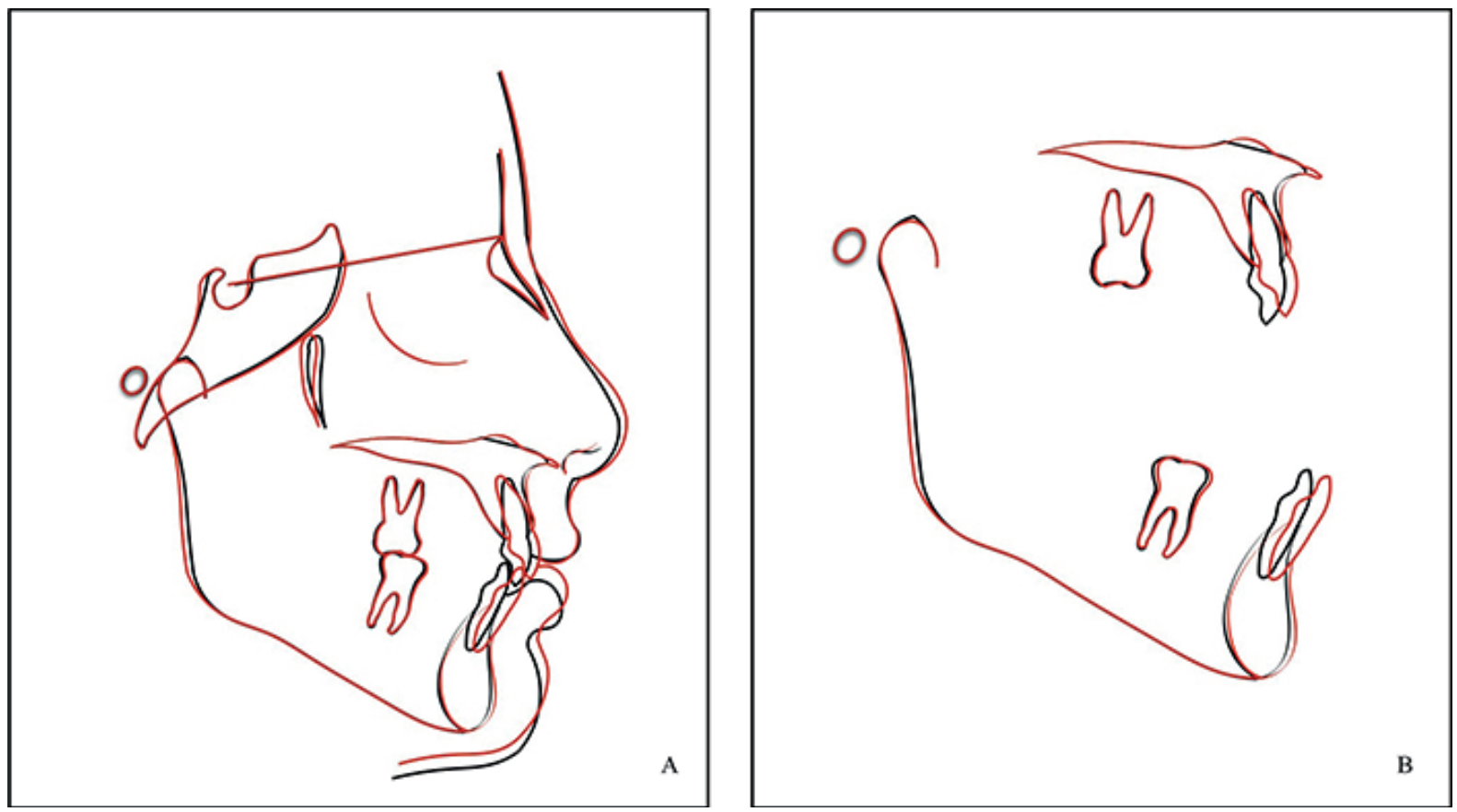

Figura 12. Superposición total (A) y superposición parcial (B). Trazado inicial en color negro y trazado final en color rojo

panorámica final muestra un correcto paralelismo de las raíces y no se evidencian signos radiográficos de reabsorción radicular (Figura 13). Los resultados del tratamiento mostraron una adecuada oclusión y estabilidad luego de dos años de seguimiento (Figura 14).

\section{Discusión}

Existen algunos principios que deben ser considerados durante el movimiento de intrusión, entre ellos: el control de la magnitud de la fuerza mediante un sistema con baja proporción de carga/deflexión, empleo de sis- tema de fuerzas estáticamente determinado al elegir un punto de contacto anterior simple y evitando insertar el arco en el slot de los bracktes, una adecuada elección del punto de aplicación de la fuerza al considerar el CR del segmento a ser intruido, evitando así efectos adversos indeseados en el movimiento de los dientes, uso adecuado de una unidad de anclaje posterior ${ }^{6-8}$. El uso de fuerzas ligeras es primordial para evitar la posible reabsorción externa apical que ocurriría al aplicar magnitudes mayores a las recomendadas ${ }^{12,13}$. Almeida et al. ${ }^{14}$ demostraron que el uso de arcos de intrusión no genera 


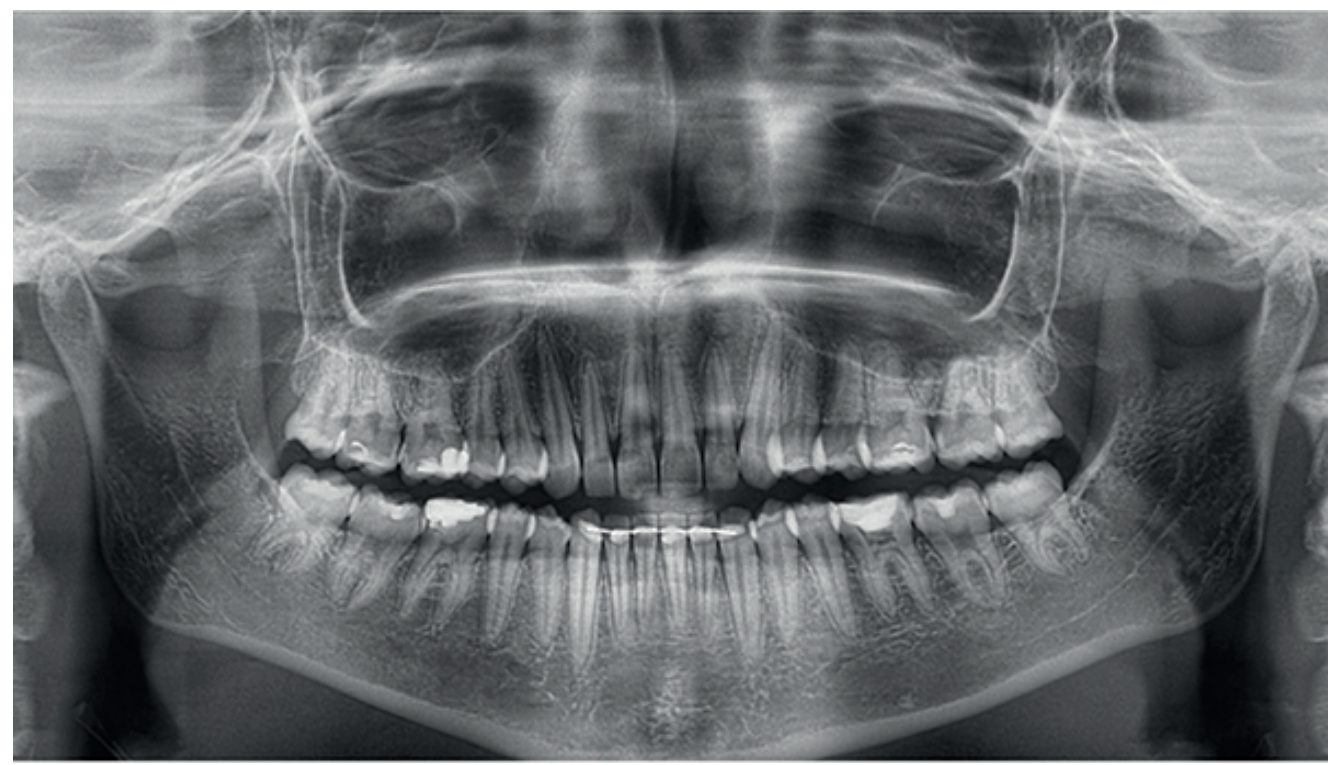

Figura 13. Radiografía panorámica final
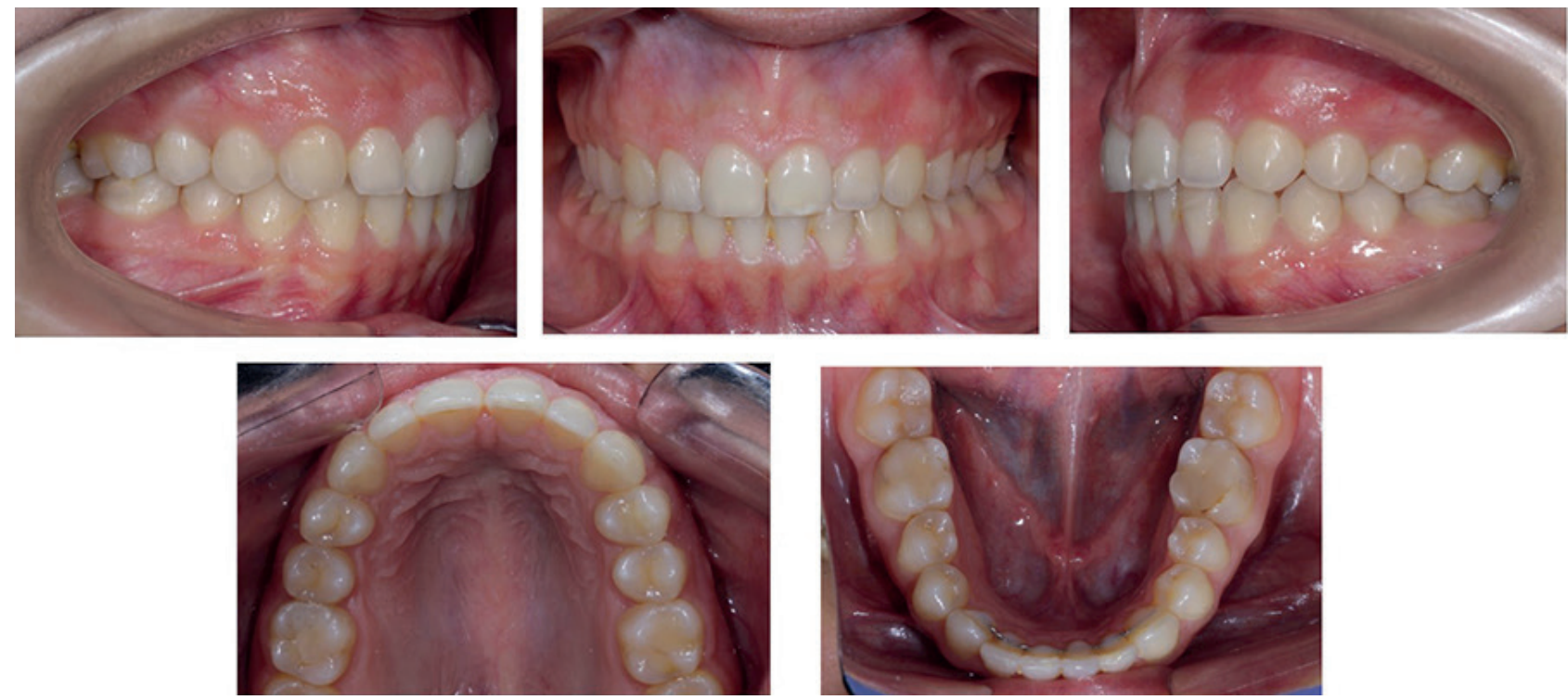

Figura 14. Fotografías intraorales luego de dos años de seguimiento

mayor reabsorción radicular externa al ser comparado con la mecánica de arco recto a través de tomografía computarizada de haz cónico.

En la literatura podemos encontrar una diversidad de abordajes para generar movimiento de intrusión de los dientes anteriores, desde las propuestas tradicionales como las de Burstone o Ricketts ${ }^{13,15}$ hasta el empleo de dispositivos de anclaje temporario (DAT's) ${ }^{16,17}$, los primeros se basan el control de magnitudes y vectores de fuerzas adecuados, generados por distintos tipos de alambre como el acero, elgiloy o TMA. El uso de DAT's también ha sido propuesto para el control vertical en las mordidas profundas, considerando que se pueden lograr intrusiones verdaderas sin efectos adversos como el movimiento recíproco de los molares a la aplicación anterior de la fuerza ${ }^{13,18}$. En caso del segmento anterior se debe considerar cuidadosamente el local de inserción con la intención de disminuir riesgos de perforación de las raíces y de reabsorción externa radicular ${ }^{19}$. En el caso presentado se seleccionó un método tradicional de intrusión en lugar del uso de DAT's aunque existía un correcto espacio entre las raíces en la zona que deberían ser colocados, debido a que el paciente no estuvo de acuerdo con esta propuesta de tratamiento. Para la confección del arco de intrusión de Burstone se empleó alambre de TMA de $0,017 \times 0,025$ " que es $40 \%$ menos rígido que el acero inoxidable, de esta forma se puede asegurar el empleo de fuerzas ligeras para la intrusión ${ }^{20}$, estimando una intrusión de los incisivos maxilares de $1,5 \mathrm{~mm}^{21}$.

Para producir un movimiento dentario de intrusión pura de los incisivos, el punto de aplicación de la fuerza debería estar ubicado por cervical de los caninos de forma bilateral, es decir distal al segmento a ser intruido, de esta manera la línea de acción de la fuerza pasaría por el CR 
de todo el segmento anterior, esta ubicación del punto de acción de la fuerza reduce la tendencia del momento a vestibularizar las coronas y palatinizar las raíces ${ }^{10}$. Sin embargo, este efecto no siempre es negativo y puede ser en algunas situaciones requerido. Particularmente, en el caso presentado se optó por aplicar la fuerza de intrusión por distal de los incisivos para generar un patrón de intrusión asociado a una ligera inclinación vestibular de los dientes con la intención de mejorar su posición en el plano sagital. Kaid y Tanielian ${ }^{22}$ compararon a través de radiografías y tomografía computarizada la inclinación axial incisal y los cambios sobre el hueso alveolar, respectivamente, luego de la intrusión con arco de Burstone y arco utilitario de Ricketts, sus resultados mostraron que los incisivos superiores tratados con arco de Burstone presentaron retroinclinación, mientras que los inferiores tratados con arco de Burstone y los superiores e inferiores tratados con arco utilitario de Ricketts mostraron proinclinación; por otro lado, la mayor pérdida de altura de hueso alveolar se observó en el proceso alveolar vestibular de los incisivos superiores tratados con arco de Burstone, hubo además disminución del grosor del hueso alveolar en todos los grupos.

Atik et al. ${ }^{23}$ evaluaron a través de tomografía computarizada de haz cónico los cambios alveolares luego de la intrusión de incisivos maxilares con arco base y con minitornillos, sus resultados mostraron que la inclinación e intrusión fue significativamente mayor en el grupo que empleó minitornillos, además observaron que, durante el tratamiento, el grosor del hueso alveolar vestibular disminuyó significativamente en este grupo

La inclusión de una unidad de anclaje posterior asociada a una liberación de fuerzas leves por parte de arco de intrusión, evitaría inclinaciones indeseadas del plano de oclusión ${ }^{8}$. Elegir sistemas de fuerza estáticamente determinados permite aplicar de manera previsible las fuerzas y momentos generados por los alambres que se mantienen estables durante la desactivación, esta característica sumada a la liberación de fuerzas leves al emplear alambres como el TMA, permite al clínico tener un mejor control de movimiento intrusivo de los dientes, al respecto se conoce que la magnitud de fuerza recomendada es de 15 a $25 \mathrm{~g}$ por diente ${ }^{6,8}$.

Conocer estas características resulta importante al momento de diseñar las mecánicas ortodónticas, haciendo que la elección de los resortes, arcos, elásticos y aparatos sean totalmente individualizados para cada caso, permitiendo controlar de manera más exacta el movimiento de los dientes. Durante el tratamiento, la paciente desarrolló una tendencia a la Clase II canina del lado izquierdo, debido al alineamiento y corrección de la mordida profunda, siendo así se indicó el uso de elásticos Clase II 3/16 asociado a un arco segmentado activado para disminuir el efecto extrusivo de los elásticos. Estos elásticos tuvieron una indicación de uso de 24 horas para poder corregir las relaciones Clase II en aproximadamente 3 meses, seguido de un doblez en " $\mathrm{z}$ " construido en un arco de acero inoxidable de 0,020 " con la intención de mejorar la inclinación de la pieza 2.3 y asegurar el asentamiento de la oclusión.

El arco de intrusión propuesto por Burstone resulta ser un dispositivo de fácil construcción y con muchas ventajas al momento de planificar el movimiento dentario de intrusión. Se deben tener conceptos claros de biomecánica como: el control de la magnitud y control del punto de aplicación de la fuerza para aprovechar de manera eficiente el potencial de este dispositivo. Además, se ha demostrado que las correcciones de mordidas profundas en pacientes adultos con el uso de arcos de intrusión son estables a largo plazo ${ }^{24}$.

El presente caso clínico permitió demostrar el uso de los conceptos biomecánicos propuestos por Burstone para la corrección de la mordida profunda, logrando resultados satisfactorios con una oclusión estable luego de dos años de seguimiento.

\section{Referencias bibliográficas}

1. Graber TM, Vanarsdall RL. Ortodoncia: Principios generales y técnicas. Buenos Aires: Editorial Médica Panamericana; 2003.

2. Proffit RW, Fields WHJr., Sarver MD. Ortodontia contemporânea. 4ta Ediçấo. São Paulo: Editora Elsevier; 2008.

3. Moyers ER. Hand book of orthodontics. 4th Edition. Chicago: Year Book Medical Publisher; 1988.

4. Brito HHA, Leite HR, Machado AW. Sobremordida exagerada: Diagnóstico e estratégias de tratamento. R Dental Press Ortodon Ortop Facial. 2009;14(3):128-157.

5. Nielsen IL. Vertical malocclusions: Etiology, development, diagnosis and some aspects of treatment. Angle Orthod. 1991;61(4):247-260.

6. Burstone CJ, Kwangchul C. The biomechanical foundation of clinical orthodontics. China: Quintessence Books; 2015.

7. Burstone CJ. Deep overbite correction by intrusion. Am J Orthod Dentofacial Orthop. 1977;72(1):1-22.

8. Burstone CJ. Biomechanics of deep overbite correction. Seminars In Orthod. 2001;7(1):26-33.

9. Sifakakis L, Pandis N, Makou M, Eliades T, Bourauel C. Forces and moments generated with various incisor intrusion systems on maxillary and mandibular anterior teeth. Angle Orthod. 2009;79(5):928-933.

10. Vanden BM, Dermaut LR, Sachdeva R, Burstone CJ. The center of resistance of anterior teeth during intrusion using the laser reflection technique and holographic interferometry. Am J Orthod. 1986;90(3):211-219.

11. Van Steenbergen E, Burstone CJ, Prahl-Andersen B, Aartman IH. The relation between the point of force application and flaring of the anterior segment. Angle Orthod. 2005;75(5):730-735.

12. Dermaut LR, De Munck A. Apical root resorption of upper incisors caused by intrusive tooth movement: A radiographic study. Am J Orthod Dentofacial Orthop. 1986;90(4):321-326. 
13. Goel P, tandor R, Agrawal KK. A comparative study of different intrusion methods and their effect on maxillary incisors. J Oral Biol Craniofac Res. 2014;4(3):186-91.

14. De Almeida MR, Marçal ASB, Fernandez TMF, Vasconcelos JB, de Almeida RR, Nanda R. A comparative study of the effect of the intrusion arch and straight wire mechanics in incisor root resorption: A randomized controlled trial. Angle Orthod. 2018;88(1):20-26.

15. Jain RK, Kumar SP, Manjula WS. Comparison of intrusion effects on maxillary incisors among mini implant anchorage, J-hook headgear and utility arch. J Clin Diagn Res. 2014;8(7):ZC21-ZC24.

16. Polat-Ozsoy O, Arman-Ozcirpici A, Veziroglu F. Miniscrews for upper incisor intrusion. Eur J Orthod. 2009;31(4):412-6.

17. Senisik NE, Turkkahraman H. Treatment effects of intrusion arches and mini-implant systems in deepbite patients. Am J Orthod Dentofacial Orthop. 2012;141(6):723-33.

18. Polat-Ozsoy O, Arman-Ozcirpici A, Vezigoglu F, Çetinsahin A. Comparison of the intrusive effects of minis- crews and utility arches. Am J Orthod Dentofacial Orthop. 2011;139(4):526-32.

19. Aras I, Tuncer AV. Comparison of anterior and posterior mini-implant-assisted maxillary incisor intrusion: Root resorption and treatment efficiency. Angle Orthod. 2016;86(5):746-52.

20. Kalra S, Tripathi T. Miniscrew supported Burstone intrusion arch. Int J Orthod Milwaukee. 2015;26(1):25-6.

21. Ng J, Major PW, Heo G, Flores-Mir C. True incisor intrusión attained during orthodontic treatment: A systematic review and meta-analysis. Am J Orthodo Dentofacial Orthop. 2005; 128(2):212-9.

22. Kaied IB, Tanielian RH. Comparative radiographic evaluation of the alveolar bone support changes after incisal intrusion. Orthodontics (Chic.). 2012;13(1):60-71.

23. Atik E, Gorucu-Coskuner H, Akarsu-Guven B, taner T. Evaluation of changes in the maxillary alveolar bone after incisor intrusion. Korean J Orthod. 2018;48(6):367-376.

24. Kale Varlik S, Onur Alpakan O, Turkoz Ç. Deepbite correction with incisor intrusion in adults: A long-term cephalometric study. Am J Orthod Dentofacial Orthop. 2013;144(3):414-9. 
